\title{
The nucleus of an atom and the periodicity of the elements
}

ABSTRACT. A study of solid matter and the application of the principle of order leads to models for the octahedral structure of the nuclei of all atoms. A model of a proton-neutron unit as a truncated tetrahedron yields interesting agreement with the eight-group periodicity of the elements. A diagram shows how the truncated tetrahedra can be fabricated from paper and these can be built up into an octahedral shape for all known elements. Other diagrams show how the "Russian doll" or "onion layers" can represent the 2, 8, 8, 18, 18 and 32 neutron-proton layers of the known elements. 48 elements are postulated for the Period 7, hence the actinides are not related to the rare-earth elements of Period 6. This is confirmed by experiment. The neutronproton unit is made up of $20 \mu$-mesons and this agrees with the high energy physics observations of protons, neutrons and mesons. $5 \mu$-mesons make a K-meson, which is another particle frequently detected in high energy physics experiments.

Nanotechnology Perceptions 11 (2015) 136-145 